\title{
Urban Traffic Jam Simulation and Prediction
}

\author{
Changbo Wang ${ }^{1, a}$, Yan Jiang ${ }^{1, b}, \mathrm{Na}$ Liu $^{1, \mathrm{c}}$ \\ ${ }^{1}$ Hubei University of Medicine, Shiyan 442000, China; \\ a'wcbqv@126.com, ${ }^{\mathrm{c}} 34703558 @ q q . c o m$ \\ Corresponding Author : ${ }^{b} 358178828 @ q q . c o m$
}

Keywords: Urban traffic flow, simulation, prediction, BML.

\begin{abstract}
As the rapid increase of traffic flow around the world, the development of the city is increasingly becoming saturated. The traffic flow theory that can guide and schedule traffic is needed. Traffic flow model based on cellular automata which is used to describe the freeway traffic flow model has been reaching maturity and applied to the actual traffic simulation system. And the traffic flow model that used to describe the city road network, referred as BML model) still remains in the stage of theoretical research. Many scholars analyze the BML model by analyzing phase-change principle and making theoretical proof. Yet the scholars have not applied the BML model into the actual urban traffic network, and intelligent scheduling and guidance. The basic BML model is improved to predict traffic congestion of the actual city road network. This study focuses on the amelioration of BML model and the numerical simulation based on this model. The main idea of this paper is: (1) the real urban traffic road network mapped to the D-BML model in accordance with a certain strategy. And the proportion of traffic volume density should be mapped to the D-BML model in accordance with of the road network in a time period. (2)D-BML model evolves in accordance with rule 184 of the cellular automaton evolution. The jam coordinate values of model grid points can be got when the model eventually enter the blocked state. (3)The severe congestion at the intersection also can be got in the next period of time. The experimental results show that the proposed D-BML model can accurately predict the blocked intersection in the road network. The first analysis of the D-BML model density of traffic is on the uniform distribution and Poisson distributions. The blockage values on the model grid are analyzed. The experiments show that as long as the initialization traffic flow density distribution determined, the clogging grid points will not change. Then engineering experiments are made. The simulation experiments of this article are about England's Birmingham City traffic network. The final experimental results are very consistent with the actual traffic clogged intersections, which strongly proved correctness and effectiveness of M-BML model.
\end{abstract}

\section{Introduction}

With the rapid development of computer technology, the cellular automata model (cellular automaton, CA) slowly raised by people [1, 2]. Cellular automata [3] is a model of discrete, which can be used to study computer science, mathematics, physics, complex scientific modeling, theoretical chemistry and microstructure. Cellular automata can also know as cellular space and surface subdivision automata, homogeneous structure, cellular structure and so on. Because all of the cell and the operation rules of traffic flow in traffic rules there is a lot of similarities, so in 1986 the earliest Cremer and Ludwig introduction to the theory of the cellular automata traffic flow theory, the thought is put forward by them to the speed, acceleration and displacement in traffic flow are regarded as discrete variables, each grid point represents a cell, in a car on the cellular automata or empty cell, for each cell to define a number of said vehicle running speed, spacing between vehicles of the displacement between the grid point indicates that the number of vehicles, all belong to update according to the same local rules. Cellular automata has the advantage that it can reflect the uncertainty of traffic flow, it can be a good description of traffic congestion, traffic accidents, car 
speed operation, the driver overreaction caused by various phenomena and so on. So the cellular automata has become the main model of traffic flow research recently [5, 6].

In establishing can accurately describe the actual road traffic characteristics of theoretical model is the main purpose of the road hand in theory research, and with the aid of traffic flow theory model to scheduling and to guide the actual traffic, traffic will be more smooth running. Last century $30 \mathrm{~s} \mathrm{has} \mathrm{a}$ lot of scholars and experts to research the traffic flow theory, the first is to study the traffic flow with mathematics method, probability method is at this time was introduced into the traffic flow theory. In 1936, Adams study and published by numerical analysis to study the traffic flow theory. In 1934, the Greenshields by actual monitoring data obtained from the state of the us highway traffic flow analysis of the relationship between linear balance speed, density, this research are still widely used today, and he also in 1947 using distribution concrete analysis of the presence of the intersection traffic flow theory research [7].

Since the $50 \mathrm{~s}$ of the last century, the automotive industry has been rapid development, to the road traffic flow is also growing, traffic flow of vehicles is more and more lost its independence is not able to free exercise and not subject to any restrictions, vehicles more and more under the influence of other vehicles on the road, and traffic flow of the probability of random phenomenon is becoming more and more low. So the method of probability theory put forward by the '30 s has been unable to meet the needs of the development of traffic flow, so it was quickly eliminated. In the fifties, the kinematics model and the car following model was applied to the study of traffic flow; In the seventy $\mathrm{s}$, research of traffic flow and fluid mechanics model of study closely link up; In the ninety s, with the development of large computer, a new model of cellular automata model by the attention of the various research fields has become a new pet. Until now, the development of the theory of traffic flow have already have, all of traffic flow has become very active. To sum up, the study of traffic flow can be divided into three categories: by describing the different methods of macro model, meso and micro model. These three models have their own advantages, and again there is a certain relationship between each other. Current based on the research of cellular automata and extension is a hotspot in the field of traffic flow, this paper is based on this, the key research based on the theory of cellular automata model of traffic flow. This chapter mainly expounds the following model, the fluid dynamics model and cellular automata model.

The paper put forward on the basis of the BML model to predict the traffic jams, the BML model was further improvements. And no scholars before using cellular automata model to predict the forecast problem of the urban traffic congestion, and also there are few scholars using the BML model to the actual traffic guidance and control. Innovation point of this article is mapped to the actual transportation network to improve the BML model and improved BML model to predict traffic congestion.

\section{The Framework of D - BML}

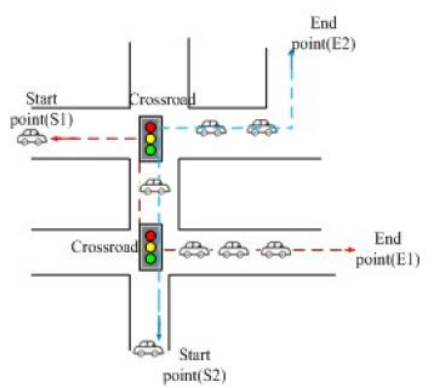

(a)

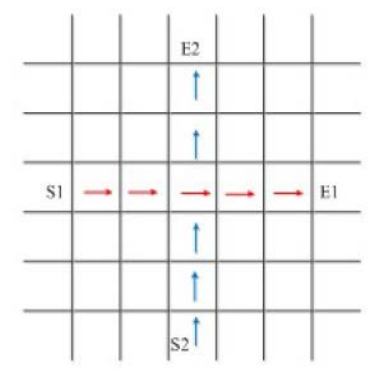

(b)

Fig. 1 The map of actual traffic road network

As shown in Fig. 1, urban traffic road network consists of seven travel sites, nine of intersection and 10 sections. To put such a complex traffic map mapped to the D - BML model, must follow certain rules. (1) From the starting point to destination point there may be multiple routes, do you want to put each route map to the BML model. We through the analysis of the above according to 
certain strategic general driving habits (driver) to select routes, to ensure the accuracy and authenticity of the model. (2) In the real city traffic, there are two on a route, the vehicle in the direction of the BML model only east and north to driving route, the route need to be mapped only once. Because in the BML model related scholars prove cause jams as horizontal and vertical direction of traffic flow interference, and parallel lines will not result in a jam. (3) In real urban traffic network, a route is made up of multiple vertical section and horizontal section, so the route map to the BML model is horizontal eastward route or perpendicular to the north of the line. For such a route, the choice in the east and north line map.

When the D - BML model initialization completes, model system D - BML model in accordance with the rules. The D - BML model operation rules is as follows. (1) The model with periodic boundary conditions, so the number of vehicles is the conservation of each line. (2) An intersection traffic lights are the rules for the time step is divided into odd time step and even the time step, in an odd number of time steps east to the vehicle may exercise, in the even time step northbound vehicles may exercise, at the time of an odd number of time steps east to moving vehicles available only when the right side of the cell to drive forward, when running vehicles in even number of time steps north to the same only when above appear empty cell to drive up. (3) The vehicle speed can only be $(0,1)$ between values.

The M - BML model running in the result of the model, the clogging of the intersection point is mapped to a real road network, as shown in figure 4 to 5 , according to the scope of the adjustment of the parameter value assumption jam is framed within the area (left). From figure can get these grid point coordinate values, such as the lower left corner of the grid point coordinate values for (12) in 2, explain that point is the intersection of 12 lines and 17 lines, corresponding to the real C5 intersection in traffic network, it can predict the real road network C5 intersection time congestion will happen in the future. But there are some complex grid points, such as coordinate values for the grid point (12, 16), its corresponding to the real network congestion intersection has two C4 and C5 intersection, then the jam is unable to determine $\mathrm{C} 4$ or $\mathrm{C} 5$, said the grid point for the conflict. And as coordinates $(15,20)$ of grid points, its response to the real network intersection is empty, call these empty points are fuzzy, in the small circle marks, namely the two route does not exist in the real network intersection.

\section{Experiments and Discussions}

The experiment of the system size is 100 x 100, so let's choose Birmingham city road network from the whole data set in vertical and horizontal intersection 100 city route for the simulation experiment. Shown in the table below, from a data set of 100 route for 10 to selected information shows that measured 10 route on April 13, 2012, 32 a period of time (i.e., eight o 'clock in the morning) traffic with AL1640, LM159, LM737 three lines of traffic. Through the analysis of the previous chapter we know that a route is made up of many a road, the traffic of different sections at the same time there is a big difference, but it does not provide the data set of this chapter of each section of each line of the traffic information in detail, by the data set may result in the imperfection of the simulation analysis of accuracy decreases. Next we map the data set of 100 lines to the $\mathrm{M}$ BML model for simulation analysis.

Table 1. The comparison of experiment results

\begin{tabular}{clll}
\hline LinkRef & Time Period & Average JT & Average Speed \\
\hline AL2 & 1 & 130.26 & 83.46 \\
AL2 & 2 & 130.38 & 83.39 \\
AL2 & 3 & 112.22 & 96.88 \\
AL2 & 6 & 118.83 & 91.49 \\
AL2 & 7 & 116.69 & 93.17 \\
AL2 & 8 & 124.79 & 87.12 \\
\hline
\end{tabular}

As shown in Table 1 , through continuous regulator can narrow the scope of the traffic jam more and more, eventually to the concrete intersection can be determined. Began when the threshold is 
very big, the congestion area is big, can be seen from the diagram congestion occurred in Birmingham city in the northeast area, further regulating valve value, when the valve value variable hours get traffic jams point is becoming more and more precise, eventually can locate to Aston station and Duddeston around the station.

\section{Summary}

With the development of modern urban traffic, urban road construction is far behind the growth of the vehicle speed, many big cities in our country face the traffic network congestion serious phenomenon such as congestion, holiday to work, on the city caused serious economic loss and environmental pollution. On the research in the field of traffic flow at present, the main also focused on the highway traffic jams and accidents, to urban traffic congestion is described in the paper there are some, but for how to ease urban traffic congestion, especially the predicted in advance is almost a blank. Used to describe the urban traffic model at present is mainly the BML model based on cellular automata, but this model rules, many scholars only for basic BML model phase change simulation demonstration or is on them to join the various realistic factors, such as the BML model considering two-way traffic flow and so on. No scholars relate BML model and actual traffic, make a guidance role model of the system. This article is aimed at this shortage, and through the BML model was improved, through the simulation of improved BML model to predict the time and place of real traffic network intersection.

Through the experimental simulation results we can be summarized as follows: (1) The first step analysis by simulation can get real traffic congestion areas, and the experimental area in the future and the actual traffic flow simulation with the traffic situation in the period of fully meet. (2) The second step is to lock in a small range that is accurate to the actual intersection, and verify its compared with the actual road traffic jams, get forecast is highly efficient, have very good guiding significance to actual traffic. (3) The third step is to model each step for a period of time after operation, and comparison between the results and the actual traffic matching rate is very high. Further proves the practicability of improved BML model proposed in this paper.

\section{References}

[1] Gao Z Y, Li K P, Li X G, et al. Scaling laws of the network traffic flow[J]. Physica A: Statistical Mechanics and its Applications, 2007, 380: 577-584.

[2] Nagatani T. Anisotropic effect on jamming transition in traffic-flow model[J]. Journal of the Physics Society Japan, 1993, 62(8): 2656-2662.

[3] Fukui M, Oikawa H, Ishibashi Y. Flow of cars crossing with unequal velocities in a two-dimensional cellular automaton model[J]. Journal of the Physical Society of Japan, 1996, 65(8): 2514-2517.

[4] Cuesta J A, Martínez F C, Molera J M, et al. Phase transitions in two-dimensional traffic-flow models[J]. Physical Review E, 1993, 48(6): R4175-R4178.

[5] Nagatani T. Effect of jam-avoiding turn on jamming transition in two-dimensional traffic flow model[J]. Journal of the Physical Society of Japan, 1994, 63(4): 1228-1231.

[6] Nagatani T. Self-organization in 2D traffic flow model with jam-avoiding drive[J]. Journal of the Physical Society of Japan, 1995, 64(4): 1421-1430.

[7] Gu G Q, Chung K H, Hui P M. Two-dimensional traffic flow problems in inhomogeneous lattices[J]. Physica A: Statistical Mechanics and its Applications, 1995, 217(3): 339-347. 